\title{
Aripiprazole, an Antipsychotic and Partial Dopamine Agonist, Inhibits Cancer Stem Cells and Reverses Chemoresistance
}

\author{
SHUHEI SUZUKI ${ }^{1,2}$, MASASHI OKADA ${ }^{1}$, KENTA KURAMOTO $^{1}$, HIROYUKI TAKEDA $^{1,2}$, \\ HIROTSUGU SAKAKI ${ }^{1,3}$, HIKARU WATARAI ${ }^{1,4}$, TOMOMI SANOMACHI ${ }^{1}$, \\ SHIZUKA SEINO ${ }^{1,5}$, TAKASHI YOSHIOKA ${ }^{2}$ and CHIFUMI KITANAKA ${ }^{1,5}$ \\ Departments of ${ }^{1}$ Molecular Cancer Science, ${ }^{2}$ Clinical Oncology, ${ }^{3}$ Obstetrics and Gynecology and \\ ${ }^{4}$ Second Department of Surgery, Yamagata University School of Medicine, Yamagata, Japan; \\ ${ }^{5}$ Research Institute for Promotion of Medical Sciences, Yamagata University Faculty of Medicine, Yamagata, Japan
}

\begin{abstract}
Background: There is a growing interest in repurposing antipsychotic dopamine antagonists for cancer treatment; however, antipsychotics are often associated with an increased risk of fatal events. The anticancer activities of aripiprazole, an antipsychotic drug with partial dopamine agonist activity and an excellent safety profile, remain unknown. Materials and Methods: The effects of aripiprazole alone or in combination with chemotherapeutic agents on the growth, sphere-forming ability and stem cell/differentiation/ chemoresistance marker expression of cancer stem cells, serumcultured cancer cells from which they were derived, and normal cells were examined. Results: At concentrations non-toxic to normal cells, aripiprazole inhibited the growth of serum-cultured cancer cells and cancer stem cells. Furthermore, aripiprazole induced differentiation and inhibited sphere formation, as well as stem cell marker expression of cancer stem cells while inhibiting their survivin expression and sensitizing them to chemotherapeutic agents. Conclusion: Repurposing aripiprazole as an anticancer stem cell drug may merit further consideration.
\end{abstract}

Thioridazine is an antipsychotic that has been used clinically in the treatment of schizophrenia and other psychoses (1). Intriguingly, a growing body of evidence indicates that thioridazine exhibits multifaceted anticancer activities in a variety of cancer cells (2). Not only does thioridazine inhibit the growth of cells derived from diverse human cancers but it can also enhance the effects of chemotherapeutic agents

Correspondence to: Masashi Okada and Chifumi Kitanaka, Department of Molecular Cancer Science, Yamagata University School of Medicine, Yamagata, 990-9585, Japan. Tel: +81 236285212, Fax: +81236285215, e-mail: m-okada@med.id.yamagata-u.ac.jp, ckitanak@med.id.yamagata-u.ac.jp

Key Words: Drug repositioning, psychiatric disorders, mental illnesses, self-renewal capacity, chemotherapy, drug resistance. by reversing multi-drug resistance (2). Moreover, thioridazine has been shown to selectively target cancer stem cells and differentiate them into non-cancer stem cells incapable of initiating tumors (3). These lines of evidence provide a strong impetus to develop this antipsychotic as a novel anticancer drug; however, thioridazine is not without serious adverse effects. Indeed, thioridazine has been associated with torsade de pointes, a potentially fatal arrhythmia (1), and a recent meta-analysis demonstrated that the risk of sudden cardiac death and sudden unexpected death was significantly increased in thioridazine users, with the risk being the highest for thioridazine among the antipsychotics surveyed in the study (4). Apparently, such life-threatening adverse effects of thioridazine would pose a substantial setback for its repurposing as an anticancer drug.

Aripiprazole, an antipsychotic widely used in the treatment of schizophrenia and related disorders, is unique in that it acts as a high-affinity partial agonist at dopamine D2 receptors (5). Aripiprazole is also characterized by its excellent safety profile $(5,6)$; thus, its use is now being considered in oncology areas for the treatment of delirium in hospitalized cancer patients (7) and morphine-induced side-effects, including emesis $(8,9)$. To date, there has been no study demonstrating an anticancer activity of aripiprazole. However, similarly to thioridazine, which reportedly exerts its anti-cancer stem cell activity via antagonism of D2-family dopamine receptors (3), aripiprazole could also act as an antagonist at D2 receptors depending on the level of endogenous receptor activation (5). We, therefore, undertook this study to test the hypothesis that aripiprazole might have anticancer activities, in particular an anti-cancer stem cell activity, similarly to thioridazine.

\section{Materials and Methods}

Antibodies and reagents. Anti-Sox2 (\#3579), anti-Nanog (\#4903), anti-E-cadherin (\#3195), anti-glial fibrillary acidic protein (GFAP) (\#3670) and anti-survivin (\#2808) antibodies were purchased from 
A

\section{IMR90}

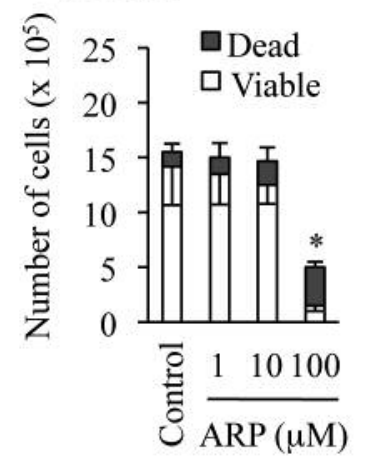

$\mathrm{C}$

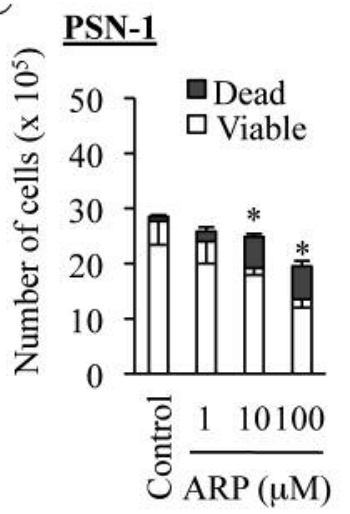

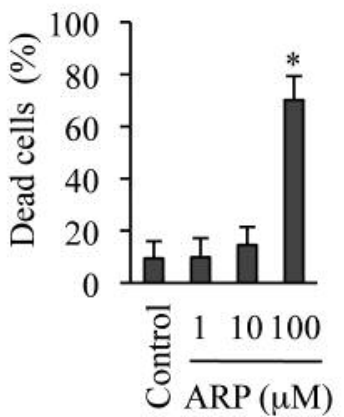

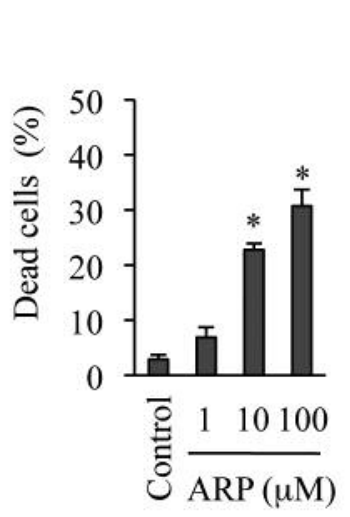

B

PANC-1
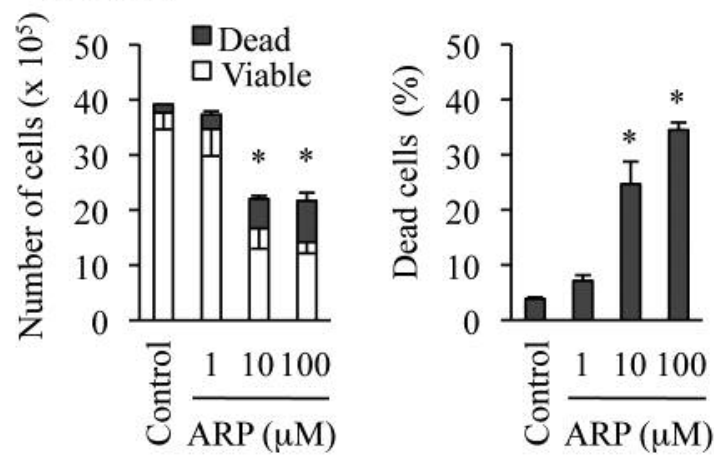

$\mathrm{D}$

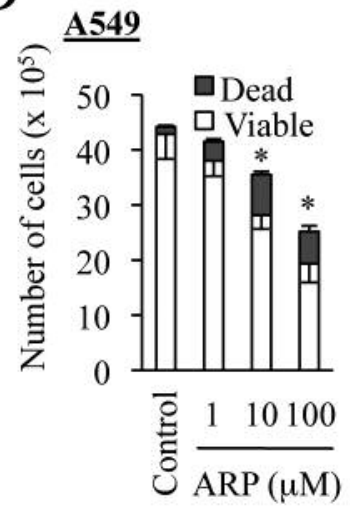

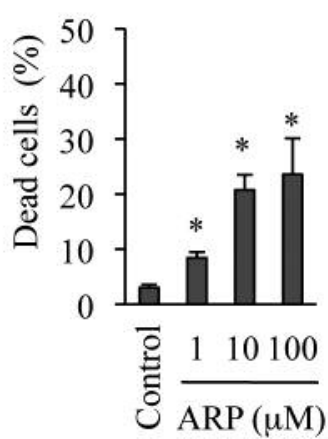

Figure 1. Aripiprazole selectively induces cell death and inhibits the growth of serum-cultured cancer cells. IMR90 normal human fibroblasts (A) and human cancer cell lines PANC-1 (B), PSN-1 (C) and A549 (D) were treated without (Control) or with the indicated concentrations of aripiprazole (ARP) for 3 days, after which the numbers of viable and dead cells (left panels), as well as the percentage of dead cells (right panels), were determined. Values in the graphs represent the means $\pm S D$ from triplicate samples of a representative experiment repeated with similar results. $* p<0.05$.

Cell Signaling Technology, Inc. (Beverly, MA, USA). Anti- $\beta$-actin (A1978) was from Sigma (St. Luis, MO, USA). Anti-CD133 (W6B3C1) was from Miltenyi Biotech (Bergisch Gladbach, Germany). 5-fluorouracil (5-FU) and gemcitabine were from Sigma and were dissolved in DMSO to prepare $200 \mathrm{mM}$ and $1 \mathrm{mM}$ stock solutions, respectively. Aripiprazole and cisplatin were from Wako Pure Chemical Industries, Ltd. (Osaka, Japan) and dissolved in DMSO to prepare a $10 \mathrm{mM}$ stock solution for each.

Cell culture. The human non-small cell lung cancer (NSCLC) cell line A549 was obtained from the Riken BioResource Center (Tsukuba, Japan). The human pancreatic cancer cell line PANC-1 was from Cell Resource Center for Biomedical Research, Institute of Development, Aging and Cancer, Tohoku University (Sendai, Japan). PSN-1 was a kind gift from Dr. Teruhiko Yoshida at National Cancer Center Research Institute (10). These cell lines were cultured in DMEM/F12 supplemented with $10 \%$ fetal bovine serum (FBS) and 100 units $/ \mathrm{ml}$ penicillin and $100 \mu \mathrm{g} / \mathrm{ml}$ streptomycin. The establishment of cancer stem cells used in this study (PANC-1 CSLC, PSN-1 CSLC, A549 CSLC and GS-Y03) has been described elsewhere (11-16). These cancer stem cells were maintained under the monolayer stem cell culture condition as previously described (11-16). Briefly, cells were cultured on collagen-I-coated dishes (IWAKI, Tokyo, Japan) in the stem cell culture medium (DMEM/F12 medium supplemented with 1\% B27 (Thermo Fisher Scientific, Waltham, MA, USA), $20 \mathrm{ng} / \mathrm{ml}$ epidermal growth factor (EGF) and fibroblast growth factor 2 (FGF2) (Peprotech, Inc., Rocky Hill, NJ, USA), D-(+)-glucose (final concentration: $26.2 \mathrm{mM}$ ), L-glutamine (final concentration: $4.5 \mathrm{mM}), 100 \mathrm{units} / \mathrm{ml}$ penicillin and $100 \mu \mathrm{g} / \mathrm{ml}$ streptomycin). Stem cell culture medium was changed every 3 days and EGF and FGF2 were added to the culture medium every day. Normal human IMR90 fetal lung fibroblasts were obtained from American Type Culture Collection (ATCC, Manassas, VA, USA) and cultured in DMEM/F12 supplemented with $10 \%$ FBS and 100 units/ml penicillin and $100 \mu \mathrm{g} / \mathrm{ml}$ streptomycin. All IMR90 experiments were done using low passage number (less than 9) cells. Rat cortical neural stem cells were purchased from R\&D systems (Minneapolis, MN, USA) and cultured in Geltrex coated plates (Thermo Fisher Scientific) in the stem cell culture medium. All rat cortical stem cell experiments were performed using low passage number (less than 9) cells. The authenticity of A549 CSLC, PANC-1 CSLC and PSN-1 CSLC as cancer stem cell lines derived from the respective original cell lines 
A

PANC-1 CSLC

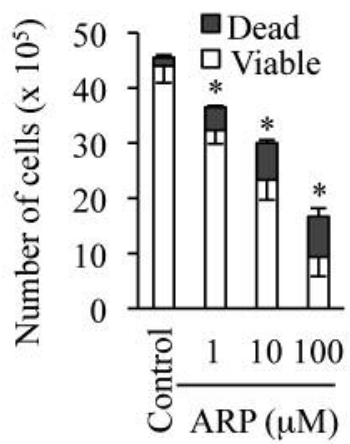

C

\section{A549 CSLC}

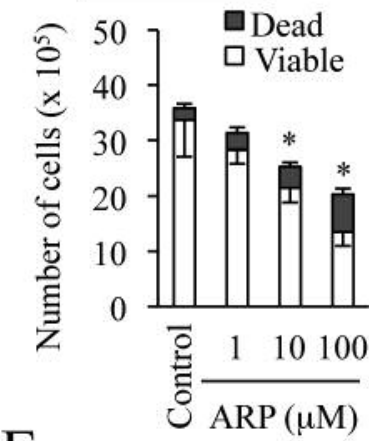

B

\section{PSN-1 CSLC}

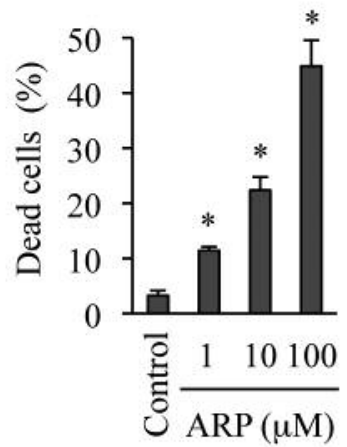

D

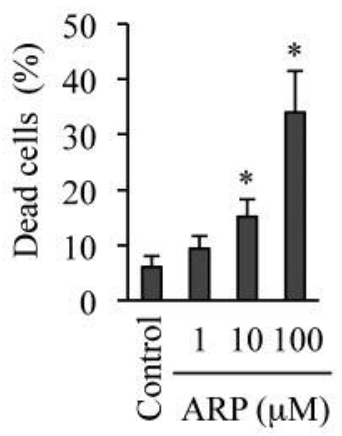

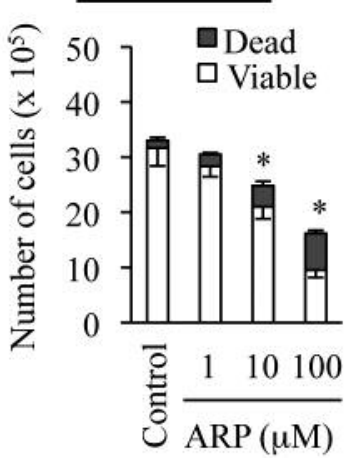

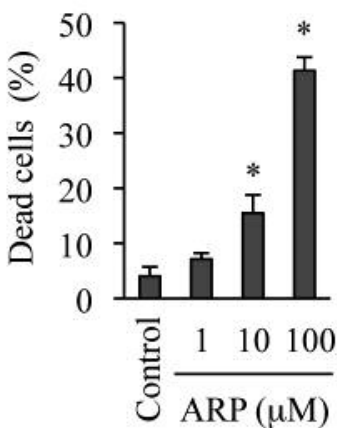

\section{GS-Y03}
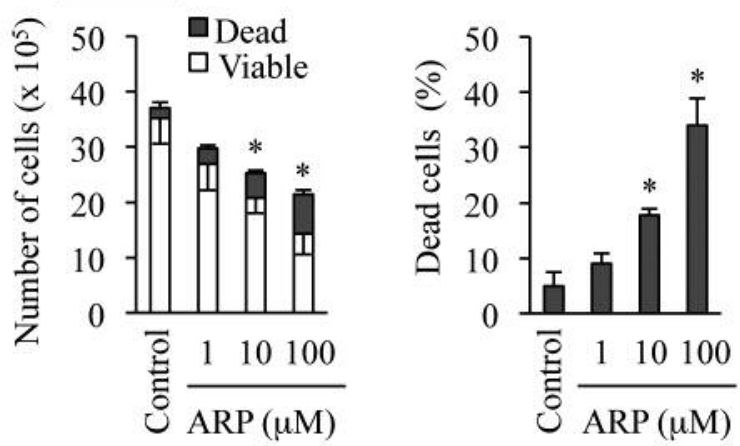

E

\section{Rat cortical neural stem cell}
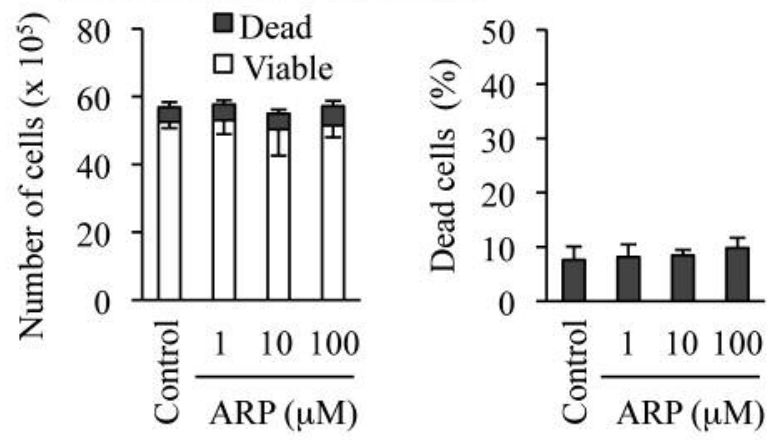

Figure 2. Aripiprazole selectively induces cell death and inhibits the growth of cancer stem cells. Cancer stem cell lines PANC-1 CSLC (A), PSN1 CSLC (B), A549 CSLC (C) and GS-Y03 (D), as well as rat cortical neural stem cells $(E)$, were treated without (Control) or with the indicated concentrations of aripiprazole (ARP) for 3 days, after which the numbers of viable and dead cells (left panels), as well as the percentage of dead cells (right panels), were determined. Values in the graphs represent the means $\pm S D$ from triplicate samples of a representative experiment repeated with similar results. ${ }^{*} p<0.05$.

was verified by genotyping short tandem repeat (STR) loci (BioSynthesis, Inc., Lewisville, TX, USA) followed by comparison to the ATCC STR Database for Human Cell Lines.

Cell viability assay. Viable and dead cells were identified by their ability and inability to exclude vital dyes, respectively (11). Briefly, cells were stained with $0.2 \%$ trypan blue and the numbers of viable and dead cells were determined using a hemocytometer. The percentage of dead cells was defined as $100 \times$ 'the number of dead cells'/('the number of viable cells' + 'the number of dead cells').

Sphere formation assay. After dissociation, single cells were serially diluted in the stem cell culture medium and seeded into non-coated 96-well plates such that each well contained a single cell. Wells 
A
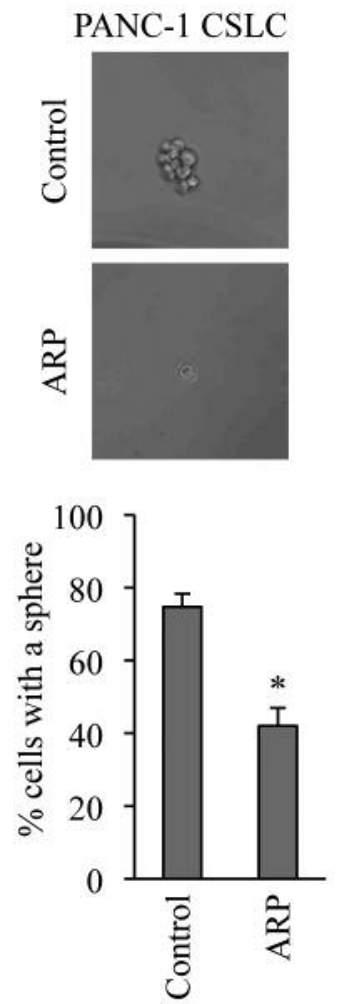

B

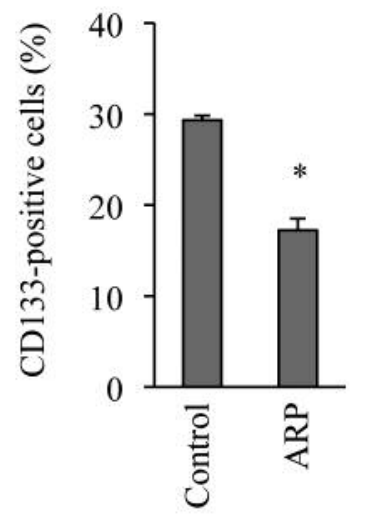

PSN-1 CSLC
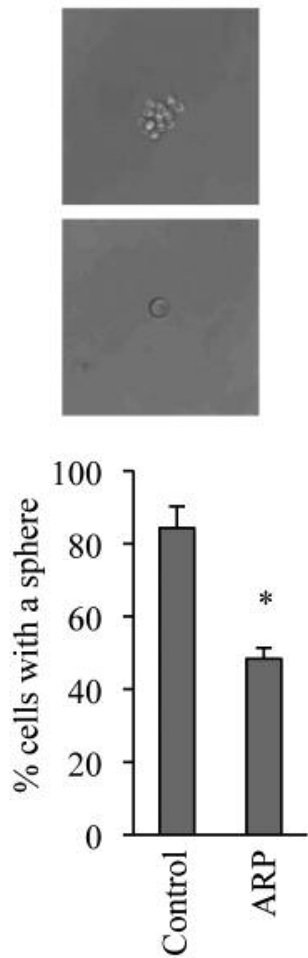

PSN-1 CSLC

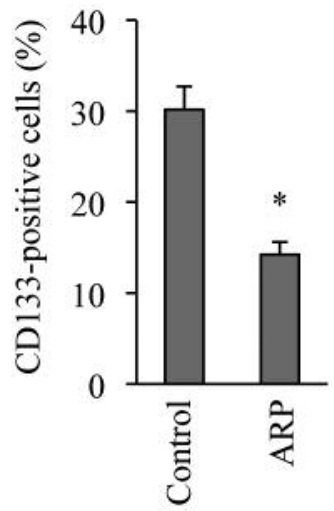

A549 CSLC
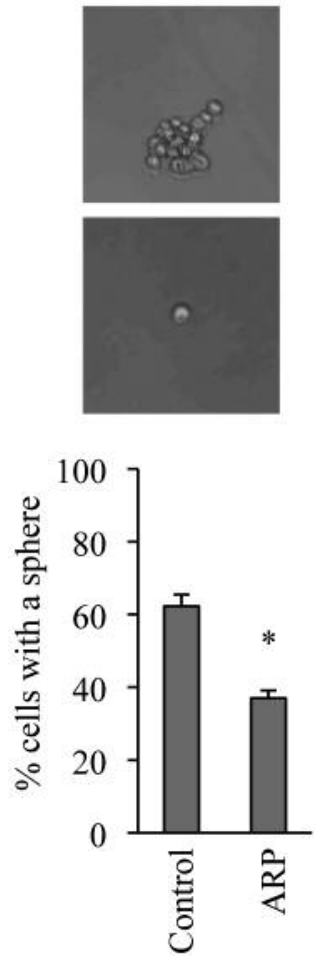

A549 CSLC

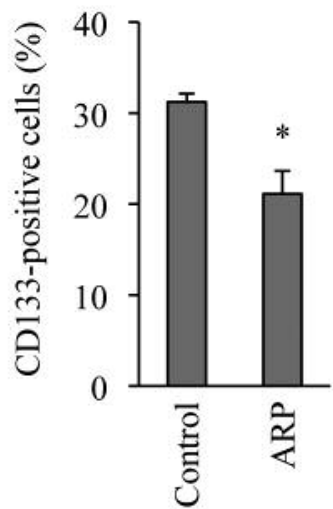

GS-Y03
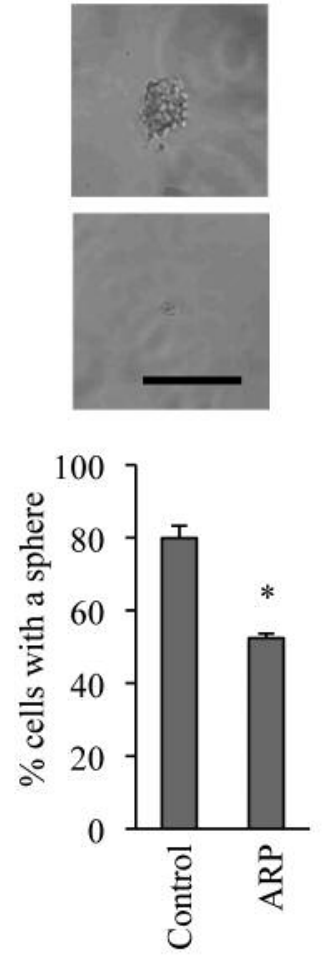

$\underline{\text { GS-Y03 }}$

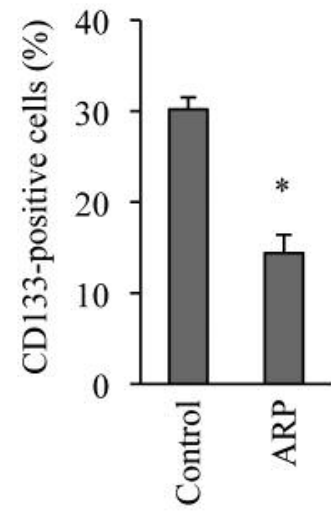

PANC-1 CSLC

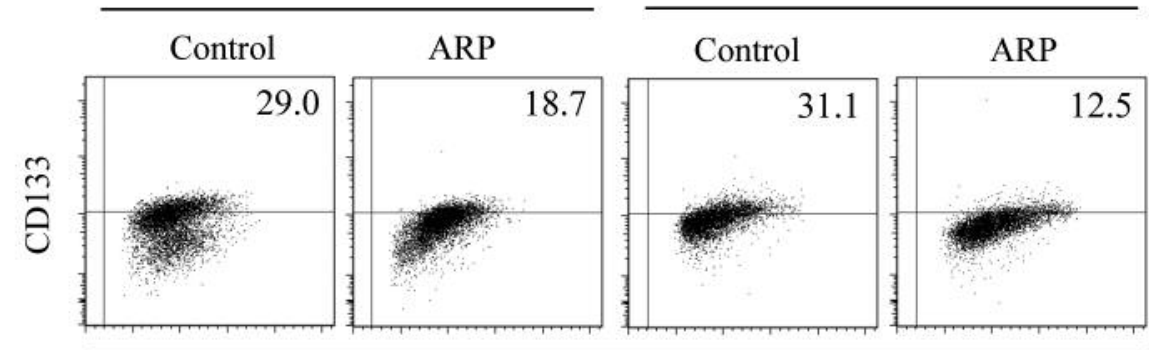

Forward Scatter 

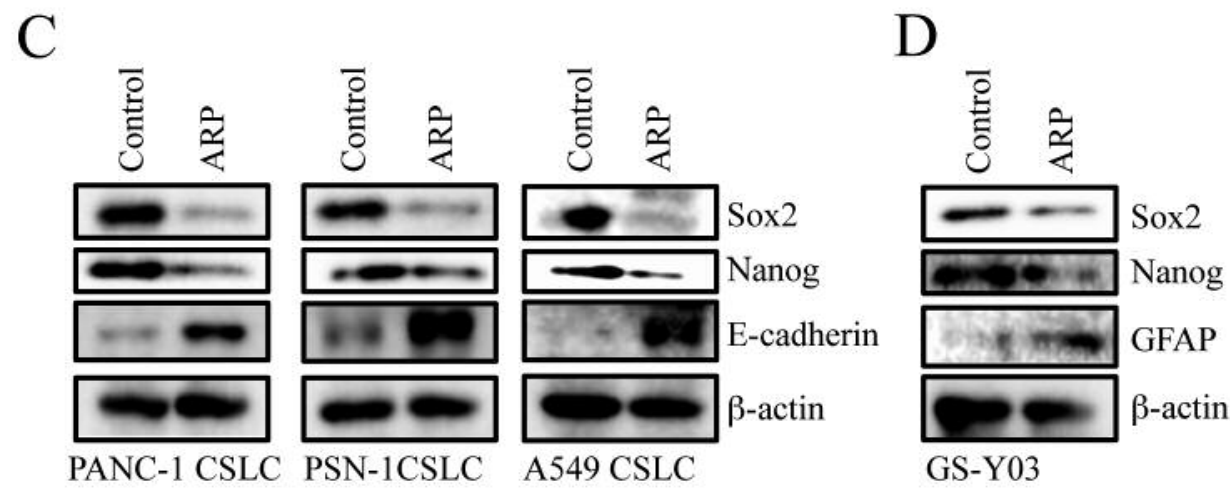

Figure 3. Aripiprazole induces the differentiation of cancer stem cells while inhibiting their sphere-forming ability and stem cell marker expression. (A) The indicated cancer stem cells treated without (Control) or with aripiprazole (ARP, $10 \mu M)$ for 3 days were subjected to a sphere formation assay in the absence of ARP. The graphs show the percentage of the wells in which a tumorsphere was formed from a single cell. Top panels show photographs of the representative wells. Scale bar, $200 \mu \mathrm{m}$. (B) Cells treated as in (A) were subjected to flow cytometry analysis for the cell surface expression of CD133. The graphs show the percentage of CD133-positive cells (upper panels). Representative flow cytometry plots together with the percentage of CD133-positive cells are shown (lower panels). (C,D) Cells treated as in (A) were subjected to immunoblot analysis for the expression of the indicated proteins. In $(A)$ and $(B)$, values in the graphs represent the means $+S D$ from 3 independent experiments. ${ }^{*} p<0.05$.

containing a single viable cell were marked under a phase-contrast microscope on the next day and, 1 week after seeding, the percentage of marked wells with a sphere relative to the total number of marked wells was determined $(12,14)$.

Immunoblot analysis. Cells were washed with ice-cold PBS and lysed in radioimmunoprecipitation assay (RIPA) buffer $(10 \mathrm{mM}$ Tris- $\mathrm{HCl}$ ( $\mathrm{pH} 7.4$ ), $0.1 \%$ SDS, $0.1 \%$ sodium deoxycholate, $1 \%$ NP$40,150 \mathrm{mM} \mathrm{NaCl}, 1 \mathrm{mM}$ EDTA, $1.5 \mathrm{mM} \mathrm{Na}_{3} \mathrm{VO}_{4}, 10 \mathrm{mM} \mathrm{NaF}, 10$ $\mathrm{mM}$ sodium pyrophosphate, $10 \mathrm{mM}$ sodium $\beta$-glycerophosphate and $1 \%$ protease inhibitor cocktail set III (Sigma). After centrifugation for $10 \mathrm{~min}$ at $14,000 \times \mathrm{g}$ at $4^{\circ} \mathrm{C}$, the supernatants were harvested as the cell lysates and protein concentration was determined by the BCA protein assay kit (Pierce Biotechnology, Inc., Rockford, IL, USA). Cell lysates containing equal amounts of protein were separated by sodium dodecyl sulfate-polyacrylamide gel electrophoresis (SDS-PAGE) and transferred to polyvinylidene difluoride membranes. The membrane was probed with a primary antibody and then with an appropriate horseradish peroxidase (HRP)-conjugated secondary antibody, according to the protocol recommended by the manufacturer of each antibody. Immunoreactive bands were visualized using Immobilon Western Chemiluminescent HRP Substrate (Millipore, Billerica, MA, USA).

Flow cytometry analysis. Flow cytometry analysis was conducted as previously described (12). In brief, dissociated cells were washed with ice-cold PBS, fixed with $4 \%$ (w/v) paraformaldehyde for 10 $\mathrm{min}$ at room temperature (RT) and washed again with ice-cold PBS The cells were blocked in FCM buffer $(0.5 \%(\mathrm{w} / \mathrm{v})$ bovine serum albumin, $0.1 \%(\mathrm{w} / \mathrm{v}) \mathrm{NaN}_{3}$ in PBS) for $1 \mathrm{~h}$, followed by three PBS rinses and further incubation with an anti-CD133 antibody in the FCM buffer overnight at $4^{\circ} \mathrm{C}$ and then with the Alexa Fluor ${ }^{\circledR} 488$ goat anti-mouse IgG (Thermo Fisher Scientific) for another $1 \mathrm{~h}$ at RT. Gating for single cells was established using forward scatter in the isotype control samples. The isotype control samples were used to establish a gate in the fluorescein isothiocyanate (FITC) channel. Cells showing a signal for CD133 above the gate established by the isotype control were deemed CD133-positive. All flow cytometry analysis experiments were run on the FACSCanto ${ }^{\mathrm{TM}}$ II Flow Cytometer (BD Biosciences, Franklin Lakes, NJ, USA). The data were analyzed by FlowJo software, version 7.6.5 (Treestar Inc., Ashland, OR, USA).

Statistical analysis. Results are expressed as the means and standard deviation (SD). The differences were compared using the 2-tailed Student's $t$-test. $p$-Values $<0.05$ were considered statistically significant and indicated with asterisks.

\section{Results}

Aripiprazole exhibits selective cytotoxic and growthinhibitory activity against both serum-cultured cancer cells and cancer stem cells. To determine the range of concentrations of aripiprazole that are not toxic to nonneoplastic cells, we first examined the viability of IMR90 normal human fibroblasts after treating them with different concentrations of aripiprazole. The results showed that IMR90 tolerated aripiprazole up to $10 \mu \mathrm{M}$ (Figure 1A). We then examined whether aripiprazole had anticancer effects. To this end, we treated three serum-cultured, human cancer cell lines (PANC-1 and PSN-1, pancreatic cancer cell lines; A549, non-small cell lung cancer cell line) with aripiprazole. The results showed that aripiprazole treatment induced cell death and accordingly decreased the number of viable cells in all three cell lines examined, suggesting that aripiprazole may have selective cytotoxic activity against neoplastic cells (Figure 1B-D). We next investigated 
whether aripiprazole had growth inhibitory effects on cancer stem cells derived from these cell lines. When the cancer stem cells derived from the cell lines (PANC-1 CSLC, PSN1 CSLC, A549 CSLC) were treated with aripiprazole at 10 $\mu \mathrm{M}$, cell growth was significantly inhibited accompanied by an increase in cell death, indicating that the cancer stem cells were also sensitive to the growth inhibitory effect of aripiprazole (Figure 2A-C). Thus, the results suggest that aripiprazole may have selective cytotoxic and growthinhibitory activity against both serum-cultured cancer cells and cancer stem cells. We also examined whether the cytotoxic and growth-inhibitory activity of aripiprazole is unique to cancer stem cells and spares normal stem cells. To this end, we treated glioma stem cells and neural stem cells with different concentrations of aripiprazole. Aripiprazole efficiently induced cell death and inhibited the growth of glioma stem cells (Figure 2D), whereas it failed to do so in neural stem cells within the range of concentrations examined (Figure 2E). Thus, aripiprazole may exert its cytotoxic and growth-inhibitory activity specifically in neoplastic stem cells.

Aripiprazole induces the differentiation of cancer stem cells into non-cancer stem cells. Prompted by the previous observation that thioridazine, an antipsychotic that acts as an antagonist at D2-family dopamine receptors, drives the differentiation of cancer stem cells into non-cancer stem cells (3), we next examined whether aripiprazole also has such a differentiation-inducing effect on cancer stem cells. To this end, we subjected cancer stem cells that survived aripiprazole treatment to sphere formation assay to evaluate whether the cells had lost their stem cell properties. The results indicated that cancer stem cells that were viable after aripiprazole treatment formed significantly less spheres in the absence of aripiprazole than those after control treatment (Figure 3A), suggesting that aripiprazole reduces the sphereforming ability of cancer stem cells surviving aripiprazole treatment. To determine whether the loss of the sphereforming ability is actually a reflection of loss of their stem cell properties, we examined the effect of aripiprazole on stem cell marker expression. Aripiprazole treatment decreased cell surface expression of CD133 (Figure 3B), as well as the expression of stem cell transcription factors Sox2 and Nanog (Figure 3C and D), in support of the idea that aripiprazole impairs self-renewal of cancer stem cells. Finally, we investigated whether aripiprazole has a differentiation-inducing effect on cancer stem cells. Aripiprazole increased the expression of E-cadherin and GFAP, markers of epithelial and astrocytic differentiation, in cancer stem cells of epithelial (Figure 3C) and astrocytic (Figure 3D) origin, respectively. Together, these results demonstrate that aripiprazole induces the differentiation of cancer stem cells into non-cancer stem cells.
Aripiprazole decreases survivin expression and sensitizes cancer stem cells to chemotherapeutic agents. Chemoresistance has often been associated with cancer stem cells and also considered a major obstacle to the elimination of cancer stem cells (17). We, therefore, asked next whether aripiprazole could reverse the chemoresistance of cancer stem cells. Treatment of cancer stem cells with the combination of aripiprazole and gemcitabine (Figure 4A) or 5-FU (Figure 4B) resulted in enhanced growth inhibition and cell death compared to either treatment alone, indicating that aripiprazole sensitized cancer stem cells to these chemotherapeutic agents. Furthermore, the combination of aripiprazole and cisplatin more effectively inhibited growth and induced death of A549 CSLC lung cancer stem cells than either alone (Figure 4C). Thus, aripiprazole sensitized cancer stem cells to multiple drugs. To explore the mechanism underlying aripiprazole-mediated sensitization to chemotherapeutic agents, we examined the impact of aripiprazole on the expression of molecules that have been implicated in multi-drug resistance of cancer cells (18). Among the molecules examined, we found that the expression of survivin was consistently decreased upon aripiprazole treatment in all cancer stem cells used in this study (Figure 5), suggesting that aripiprazole-mediated down-regulation of survivin expression may have a role in the mechanism by which aripiprazole sensitizes cancer stem cells to chemotherapeutic agents.

\section{Discussion}

In this study, we showed that aripiprazole induces cell death and inhibits the growth of not only serum-cultured (i.e., most likely non-stem) cancer cells, but also cancer stem cells while sparing normal cells, including tissue stem cells. We also provided lines of evidence that aripiprazole promotes loss of stem cell properties and drives the differentiation of cancer stem cells into non-cancer stem cells. Furthermore, aripiprazole inhibited survivin expression and effectively sensitized cancer stem cells to multiple chemotherapeutic agents. Altogether, these findings suggest that aripiprazole may be a promising drug candidate targeting cancer stem cells.

It remains to be seen how aripiprazole affects the cellular functions of cancer stem cells. However, given the previous observation that thioridazine exerts its anticancer stem cell activity through antagonism at dopamine D2 receptors, we are currently assuming that aripiprazole's effects observed in this study are dependent on its action as an antagonist at D2 receptors on cancer stem cells, though contribution of its action at other receptors, such as 5-hydroxytryptamine receptors, is not excluded $(5,19)$. It is also unknown how signaling pathways downstream of dopamine D2 receptors regulate the stem cell properties of cancer stem cells. Intriguingly, in this regard, our preliminary data indicated that aripiprazole prevented nuclear localization of $\beta$-catenin 
A
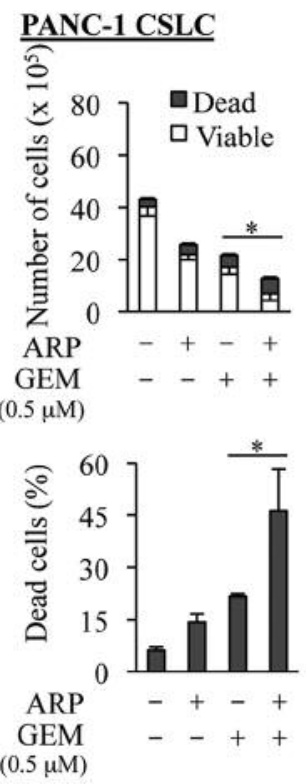

B PANC-1 CSLC

$\tilde{\sigma}$

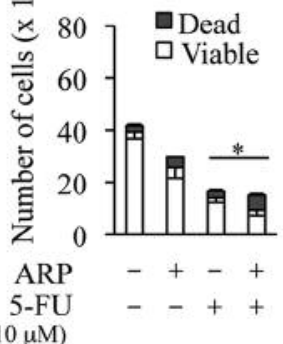

$(10 \mu \mathrm{M})$

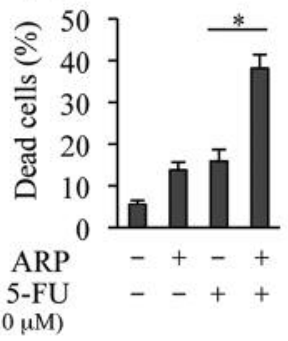

C $\stackrel{\text { A549 CSLC }}{S}$

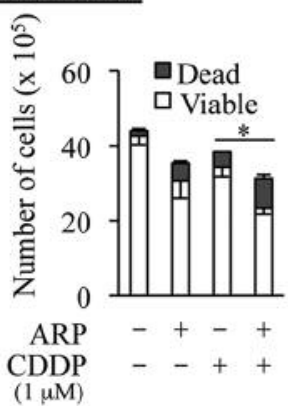

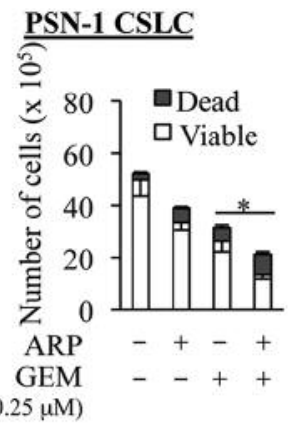

$\underline{\mathrm{A} 549 \mathrm{CSLC}}$
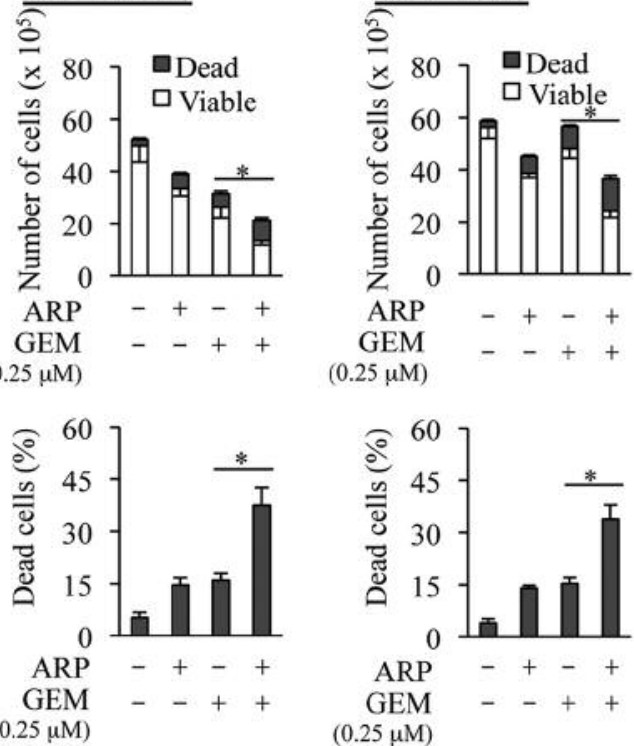

PSN-1 CSLC
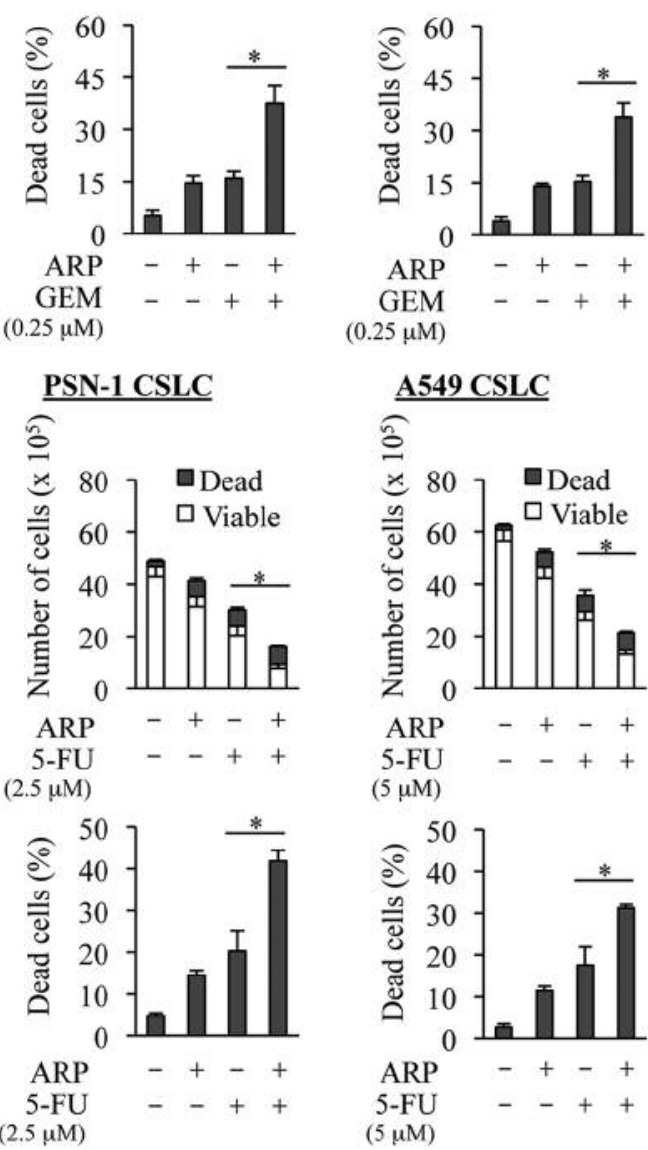

Figure 4. Aripiprazole sensitizes cancer stem cells to chemotherapeutic agents. Cells were treated with gemcitabine (GEM) (A), 5-fluorouracil (5$F U)(B)$ or cisplatin $(C D D P)(C)$ in the absence or presence of aripiprazole $(A R P, 10 \mu M)$ for 3 days, after which the numbers of viable and dead cells (upper or left panels), as well as the percentage of dead cells (lower or right panels), were determined. Values represent the means $\pm S D$ from triplicate samples of a representative experiment repeated with similar results. ${ }^{*} p<0.05$. 


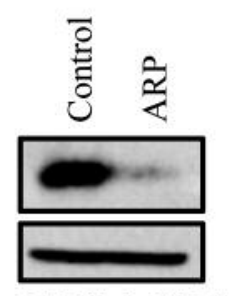

PANC-1 CSLC

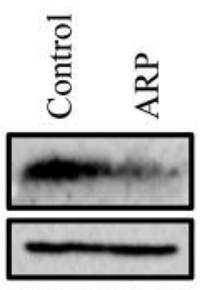

PSN-1 CSLC

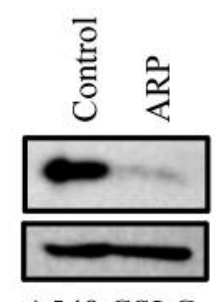

A549 CSLC

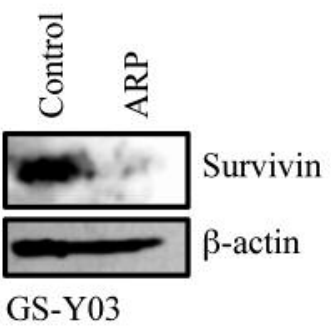

GS-Y03

Figure 5. Aripiprazole decreases survivin expression. Cells treated without (Control) or with aripiprazole (ARP, $10 \mu M)$ for 3 days were subjected to immunoblot analysis for survivin expression.

(unpublished observation), suggesting that the Wnt signaling pathway known to play an important role in the maintenance of stem cell properties may be involved therein.

We found, in this study, that aripiprazole caused a marked reduction in the expression of survivin in cancer stem cells. Since survivin is an anti-apoptotic protein overexpressed specifically in cancer cells and confers resistance to multiple chemotherapeutic agents, control of survivin expression/ function has been considered a key to reversal of multi-drug resistance of cancer cells $(18,20)$. Our results, therefore, imply that combining aripiprazole with chemotherapeutic agents might be a rational and promising approach to treating cancer. In line with such an idea, the combinations of aripiprazole and gemcitabine, 5FU or cisplatin turned out to be more effective in inhibiting the growth of cancer stem cells than either alone. Although a previous study demonstrated that thioridazine inhibits the expression of P-glycoprotein (21), our preliminary data indicated that $\mathrm{P}$-glycoprotein expression was rather increased by aripiprazole treatment in A549 CSLC cells, whereas aripiprazole did decrease its expression in PANC1 CSLC, PSN-1 CSLC and GS-Y03 cells (unpublished observation). Thus, down-regulation of P-glycoprotein may not be a major, at least not a common, mechanism by which aripiprazole sensitizes cancer stem cells to chemotherapeutic agents.

Our identification of aripiprazole as a potential anticancer stem cell drug, together with the previous report demonstrating the selective cancer stem cell-targeting effect of thioridazine (3), suggests that repurposing of antipsychotic drugs with antagonistic activity at dopamine D2 receptors may be an attractive strategy to develop novel drugs to treat cancer stem cells. In this regard, each candidate drug needs to be evaluated carefully in vitro before it is tested in preclinical animal models and clinical human studies. Indeed, we found that olanzapine, which belongs to a class of antipsychotics called multi-acting-receptor-targeted antipsychotic, had cytotoxic and differentiation-inducing activity in cancer stem cells similarly to aripiprazole. However, olanzapine did so only at concentrations toxic to normal cells in contrast to aripiprazole, precluding its further development as an anticancer stem cell drug. Another vital factor to be taken into consideration is the clinical safety information of each candidate. Although current antipsychotic medications have substantially decreased risk of extrapyramidal side-effects, they still are associated with a significant incidence of fatal events, in particular sudden cardiac death from arrhythmia $(1,4,22)$. Given the outstanding safety profile of aripiprazole, the in vitro data provided in this study apparently warrants further pursuit of aripiprazole as an anticancer stem cell drug through preclinical and clinical studies.

In conclusion, demonstrated, for the first time, that aripiprazole not only has cytotoxic and growth-inhibitory activity in cancer stem cells but also drives their differentiation into non-cancer stem cells and sensitizes them to multiple chemotherapeutic agents. Our findings, thus, provide substantial evidence with which to consider repurposing of this safe antipsychotic drug as a novel cancer stem cell-targeting agent.

\section{Conflicts of Interest}

The Authors declare no conflict of interest.

\section{Acknowledgements}

This work was supported by Grants-in-Aid for Scientific Research, for Challenging Exploratory Research, and for Young Scientists from the Ministry of Education, Culture, Sports, Science and Technology of Japan.

\section{References}

1 Fenton M, Rathbone J, Reilly J and Sultana A: Thioridazine for schizophrenia. Cochrane Database Syst Rev: CD001944, 2007.

2 Mu J, Xu H, Yang Y, Huang W, Xiao J, Li M, Tan Z, Ding Q, Zhang L, Lu J, Wu X and Liu Y: Thioridazine, an antipsychotic drug, elicits potent antitumor effects in gastric cancer. Oncol Rep 31: 2107-2114, 2014. 
3 Sachlos E, Risueno RM, Laronde S, Shapovalova Z, Lee JH, Russell J, Malig M, McNicol JD, Fiebig-Comyn A, Graham M, Levadoux-Martin M, Lee JB, Giacomelli AO, Hassell JA, Fischer-Russell D, Trus MR, Foley R, Leber B, Xenocostas A, Brown ED, Collins TJ and Bhatia M: Identification of drugs including a dopamine receptor antagonist that selectively target cancer stem cells. Cell 149: 1284-1297, 2012.

4 Salvo F, Pariente A, Shakir S, Robinson P, Arnaud M, Thomas S, Raschi E, Fourrier-Reglat A, Moore N, Sturkenboom M and Hazell On Behalf Of Investigators Of The Aritmo Consortium L: Sudden cardiac and sudden unexpected death related to antipsychotics: A meta-analysis of observational studies. Clin Pharmacol Ther 99: 306-314, 2016.

5 Burris KD, Molski TF, Xu C, Ryan E, Tottori K, Kikuchi T, Yocca FD and Molinoff PB: Aripiprazole, a novel antipsychotic, is a high-affinity partial agonist at human dopamine D2 receptors. J Pharmacol Exp Ther 302: 381-389, 2002.

6 Marder SR, McQuade RD, Stock E, Kaplita S, Marcus R, Safferman AZ, Saha A, Ali M and Iwamoto T: Aripiprazole in the treatment of schizophrenia: Safety and tolerability in shortterm, placebo-controlled trials. Schizophr Res 61: 123-136, 2003.

7 Boettger S and Breitbart W: An open trial of aripiprazole for the treatment of delirium in hospitalized cancer patients. Palliat Support Care 9: 351-357, 2011.

8 Narita M, Takei D, Shiokawa M, Tsurukawa Y, Matsushima Y, Nakamura A, Takagi S, Asato M, Ikegami D, Narita M, Amano T, Niikura K, Hashimoto K, Kuzumaki $\mathrm{N}$ and Suzuki T: Suppression of dopamine-related side effects of morphine by aripiprazole, a dopamine system stabilizer. Eur J Pharmacol 600: 105-109, 2008.

9 Shiokawa M, Narita M, Nakamura A, Kurokawa K, Inoue T and Suzuki T: Usefulness of the dopamine system-stabilizer aripiprazole for reducing morphine-induced emesis. Eur J Pharmacol 570: 108-110, 2007.

10 Yamada H, Yoshida T, Sakamoto H, Terada M and Sugimura T: Establishment of a human pancreatic adenocarcinoma cell line (PSN-1) with amplifications of both c-myc and activated c-Kiras by a point mutation. Biochem Biophys Res Commun 140: 167-173, 1986.

11 Okada M, Sato A, Shibuya K, Watanabe E, Seino S, Suzuki S, Seino M, Narita Y, Shibui S, Kayama T and Kitanaka C: JNK contributes to temozolomide resistance of stem-like glioblastoma cells via regulation of MGMT expression. Int J Oncol 44: 591599, 2014.

12 Okada M, Shibuya K, Sato A, Seino S, Suzuki S, Seino M and Kitanaka C: Targeting the K-Ras--JNK axis eliminates cancer stem-like cells and prevents pancreatic tumor formation. Oncotarget 5: 5100-5112, 2014.
13 Matsuda K, Sato A, Okada M, Shibuya K, Seino S, Suzuki K, Watanabe E, Narita Y, Shibui S, Kayama T and Kitanaka C: Targeting JNK for therapeutic depletion of stem-like glioblastoma cells. Sci Rep 2: 516, 2012.

14 Okada M, Kuramoto K, Takeda H, Watarai H, Sakaki H, Seino S, Seino M, Suzuki S and Kitanaka C: The novel JNK inhibitor AS602801 inhibits cancer stem cells in vitro and in vivo. Oncotarget 7: 27021-27032, 2016.

15 Okada M, Shibuya K, Sato A, Seino S, Watanabe E, Suzuki S, Seino $M$ and Kitanaka C: Specific role of JNK in the maintenance of the tumor-initiating capacity of A549 human non-small cell lung cancer cells. Oncol Rep 30: 1957-1964, 2013.

16 Suzuki S, Okada M, Shibuya K, Seino M, Sato A, Takeda H, Seino S, Yoshioka T and Kitanaka C: JNK suppression of chemotherapeutic agents-induced ROS confers chemoresistance on pancreatic cancer stem cells. Oncotarget 6: 458-470, 2015.

17 Zhao J: Cancer stem cells and chemoresistance: The smartest survives the raid. Pharmacol Ther 160: 145-158, 2016.

18 Fatemian T, Othman I and Chowdhury EH: Strategies and validation for siRNA-based therapeutics for the reversal of multi-drug resistance in cancer. Drug Discov Today 19: 71-78, 2014.

19 Jordan S, Koprivica V, Chen R, Tottori K, Kikuchi T and Altar CA: The antipsychotic aripiprazole is a potent, partial agonist at the human 5-HT1A receptor. Eur J Pharmacol 441: 137-140, 2002.

20 Singh N, Krishnakumar S, Kanwar RK, Cheung CH and Kanwar JR: Clinical aspects for survivin: A crucial molecule for targeting drug-resistant cancers. Drug Discov Today 20: 578-587, 2015.

21 Spengler G, Molnar J, Viveiros M and Amaral L: Thioridazine induces apoptosis of multidrug-resistant mouse lymphoma cells transfected with the human $\mathrm{ABCB} 1$ and inhibits the expression of P-glycoprotein. Anticancer Res 31: 4201-4205, 2011.

22 Ames D, Carr-Lopez SM, Gutierrez MA, Pierre JM, Rosen JA, Shakib S and Yudofsky LM: Detecting and managing adverse effects of antipsychotic medications: Current state of play. Psychiatr Clin North Am 39: 275-311, 2016.
Received September 14, 2016

Revised September26, 2016

Accepted September 27, 2016 